\title{
STATISTICAL METHODS FOR PROPERTY CALCULATION OF TEXTURED MATERIALS
}

\author{
T. D. SHERMERGOR \\ Institute of Electronic Technology, Moscow, Russia \\ (Received 30 July 1995; in final form 1 December 1995)
}

\begin{abstract}
Statistical methods for calculating the effective static and dynamic characteristics of textured microheterogeneous materials such as polycrystals, composites and rocks are considered. Effective static characteristics based on the example of the elastic modules tensor are analyzed. Effective dynamic characteristics based on the example of the dielectric permeability tensor in calculating the scattering factor, phase and group velocities of the propagation of plane electromagnetic waves are presented.
\end{abstract}

KEY WORDS: Effective static and dynamic characteristics, microinhomogeneous media, scattering, velocity of waves, texture

\section{INTRODUCTION}

One of the important problems of the mechanics and physics of solid bodies is the calculation of effective static and dynamic properties of microheterogeneous materials such as onecomponent and multicomponent polycrystals, rocks, and composites. Usually such materials consist of several components, each of which can be represented as a matrix, or as a set of separate grains. Each grain is in turn characterized by a certain form, and for polycrystals and rocks by anisotropy as well.

As a whole a macroscopic object can be also anisotropic. The reasons of anisotropy can be both a preferred orientation of nonspherical grains of components, and a particular orientation of crystallographic axes of separate crystallites. It is impossible to take into account all these factors. Therefore certain approximations are assumed. More often, especially for textures formed by ordering of crystallographic axes of separate crystallites, the approach of replacing effective values of material parameters with the average ones is accepted (Bunge, 1993). Even such kind of problem appears to be reasonably complicated, as far as orientation distribution function (ODF) calculation from experimentally measured pole figures is concerned. Thus errors of recalculation, as well as the necessity of empirical selection of the approximated function are imposed on the experimental error.

At the same time, if the anisotropy of macroscopic properties of a textured material is not small, it turns out, that the difference between the effective and average properties can be essential. Therefore a calculation of effective properties with the use of a rough approximation of the ODF is of interest for such materials. Thus the lack of information about the true ODF will be compensated by the more exact magnitudes of material parameters at the expense of the difference between the average and effective magnitude of these values. 
From various methods of calculation of the effective material characteristics the most recognition was received by the approach, based on the use of statistical methods, in particular, a random field theory (Rytov and others, 1978, Chernov, 1960, Klyatskin, 1980, Shermergor, 1977). This approach was successfully used in the theory of turbulence of a liquid (Monin and Jaglom, 1965, 1967), gas (Tatarskij, 1961) and a plasma (Ginzburg, 1967). A feature of this method is that fluctuations, caused by those or other reasons, are subject to Gauss distribution. The latter permits to use Feinman diagrams, as the even moments of the higher orders can be expressed through the moment of the second order, whereas the odd moments are zero. Another situation takes place for microheterogeneous solid media. For them the borders between separate grains turn out to be sharp, which from the mathematical point of view should be reflected by a gap of the derivative binary correlation function when passing through zero. An elementary function of this type is the $\exp (-\mathrm{r} / \mathrm{a})$, in which the parameter a plays the role of the exponential function space scale correlation.

The above dependence is typical for a completely disordered material. Obviously, for a textured material such kind of dependence can not occur. Therefore it is of interest to generalize this dependence so that it takes anisotropy into account. The appropriate approach will be considered below.

\section{THE FORMAL OUTLINE}

Among material parameters the effective properties of which are usually calculated we shall first of all note the elasticity constants, the permittivity, and the piezocoefficients. For magnetic materials effective permeability or susceptibility can be considered, however the difficulties here are that for dia- and paramagnetics the permeability differs a little from unit, whereas for ferromagnetics even in weak magnetic fields a nonlinearity of the relationship between the magnetic induction and the intensity of the magnetic field is displayed. Alongside with the effective factors of transfer - heat conductivity, electroconductivity, viscosity, and diffusion can be considered. Below, for definiteness, the effective static parameters in the case of constant elasticity are considered.

In a nonindex form of recording the connection between the pressure $\sigma$ and the deformations $\varepsilon$ has a form

$$
\sigma(\overrightarrow{\mathrm{r}})=\mathrm{c}(\overrightarrow{\mathrm{r}}) \varepsilon(\overrightarrow{\mathrm{r}})
$$

Here $\mathrm{c}$ is the tensor of elastic modules. Let us average this expression. Then we shall obtain a relation between the average pressure and the deformations

$$
\langle\sigma\rangle=\langle c \varepsilon\rangle=\mathrm{c}^{*}\langle\varepsilon\rangle
$$

Thus it is seen that the relation between the average magnitude of the fields of stress and deformations is executed through the effective tensor of modules of the elasticity $c^{*}$. Presenting all the values as the average magnitude and fluctuations

we shall obtain

$$
\sigma=\langle\sigma\rangle+\sigma^{\prime}
$$

$$
\langle\sigma\rangle=\langle c\rangle\langle\varepsilon\rangle+\left\langle c^{\prime} \varepsilon^{\prime}\right\rangle
$$


Within the framework of the linear theory there should exist a linear relationship between the fluctuation of the field of deformations and its average magnitude.

$$
\varepsilon^{\prime}=\mathrm{P}^{\prime}\langle\varepsilon\rangle
$$

Here $\mathrm{P}^{\prime}$ is some stochastic operator. Substituting (1.5) in (1.4), we receive the formal solution of the above problem:

$$
\mathrm{c}^{*}=\langle\mathrm{c}\rangle+\left\langle\mathrm{c}^{\prime} \mathrm{P}^{\prime}\right\rangle
$$

\section{THE STATIC EFFECTIVE CHARACTERISTICS}

We shall proceed from the equation of balance for microheterogeneous media

$$
\mathrm{L}_{\mathrm{ij}} \mathrm{u}_{\mathrm{j}}=-\mathrm{F}_{\mathrm{i}}
$$

and media of comparison. In the latter case we shall mark all quantities by the index c:

From (2.2) and (2.1) we find

$$
\mathrm{L}_{\mathrm{ij}}^{\mathrm{c}} \mathrm{u}_{\mathrm{j}}^{\mathrm{c}}=-\mathrm{F}_{\mathrm{i}}
$$

$$
\begin{gathered}
L^{c} u^{\prime \prime}=-L^{\prime \prime} u, \\
u^{\prime \prime} \equiv u-u^{c}, L^{\prime \prime} \equiv L-L^{c}
\end{gathered}
$$

Here a nonindex representation is used. From here, introducing the operator $\mathrm{L}^{\mathrm{c}}$ connected with Green function $\mathrm{G}^{\mathrm{c}}$

we obtain

$$
\begin{aligned}
& L^{c} G^{c}=-I \delta(\vec{r}) \\
& u=G^{c} * L^{\prime \prime} u
\end{aligned}
$$

where the asterisk designates the operation of the integrated convolution. From here, by differentiating and carrying out the symmetrisation on the indexes, we find a random part of the tensor of deformations

$$
\begin{aligned}
& \varepsilon_{\mathrm{ij}}^{\prime} \equiv \varepsilon-\langle\varepsilon\rangle=\mathrm{Q}_{\mathrm{ijk} \mid} \mathrm{c}_{\mathrm{klmn}}^{\prime} \varepsilon_{\mathrm{mn}}=\mathrm{G}_{\mathrm{i})(\mathrm{kl})(\mathrm{j}}^{\mathrm{c}} * \mathrm{c}^{\prime}{ }_{\mathrm{klmn}} \varepsilon_{\mathrm{mn}}, \\
& c^{\prime}{ }_{k l m n} \equiv c_{\text {klmn }}-\left\langle c_{\text {klmn }}\right\rangle
\end{aligned}
$$

Here $Q_{i j k l}$ is the integral operator and we assumed $c^{c} \equiv\langle c\rangle$. Its kernel is a symmetrized second derivative of the regular Green function. From here, for the tensor of deformations we obtain

$$
\varepsilon=\left(\mathrm{I}-\mathrm{Qc}^{\prime}\right)^{-1}\left\langle(\mathrm{I}-\mathrm{Qc})^{-1}\right\rangle^{-1}\langle\varepsilon\rangle
$$

Substituting this expression in the formula (1.2) for the average pressure, we find an obvious expression for the effective tensor of modules of elasticity with

$$
\langle\sigma\rangle=\langle\mathrm{c} \varepsilon\rangle=\left\langle\mathrm{c}\left(\mathrm{I}-\mathrm{Qc}^{\prime}\right)^{-1}\right\rangle\left\langle\left(\mathrm{I}-\mathrm{Qc}^{\prime}\right)^{-1}\right\rangle^{-1}\langle\varepsilon\rangle
$$




$$
\mathrm{c}^{*}=\left\langle\mathrm{c}\left(\mathrm{I}-\mathrm{Q} \mathrm{c}^{\prime}\right)^{-1}\right\rangle\left\langle\left(\mathrm{I}-\mathrm{Qc}^{\prime}\right)^{-1}\right\rangle^{-1}
$$

Expression (2.9) can be substituted in an integral operators series

$$
\left(\mathrm{I}-\mathrm{Q} \mathrm{c}^{\prime}\right)^{-1}=\Sigma\left(\mathrm{Qc}^{\prime}\right)^{\mathrm{n}}
$$

From here, being limited by the terms of the fourth order on $c^{\prime}$, we find

$$
\mathrm{c}^{*}=\langle\mathrm{c}\rangle+\left\langle\mathrm{cQc}^{\prime}\right\rangle+\left\langle\mathrm{cQc}^{\prime} \mathrm{Qc}^{\prime}\right\rangle+\left\langle\mathrm{cQc}^{\prime} \mathrm{Qc}^{\prime} \mathrm{Qc}^{\prime}\right\rangle-\left\langle\mathrm{CQc}^{\prime}\right\rangle\left\langle\mathrm{Qc}^{\prime} \mathrm{Qc}^{\prime}\right\rangle+\ldots
$$

As it is seen from the above expression, each term except the first one contains correlation functions of various orders. Usually the series is truncated after the second forum, taking into account the correlation function of the second order. This approximation is commonly called the correlation approximation. In another approach it is possible not to use the series (2.11) for the effective module, but limited singular approximation on which only a singular part of the second derivative of Green function is taken into account. The physical sense of this approximation is that the field inside the grain is assumed to be homogeneous.

The main formula of the generalized singular approximation of the theory of random functions is

$$
\mathrm{c}^{*}=\left\langle\mathrm{c}\left(\mathrm{I}-\mathrm{gc}^{\prime}\right)^{-1}\right\rangle\left\langle\left(\mathrm{I}-\mathrm{gc}^{\prime}\right)^{-1}\right\rangle^{-1}
$$

Here $g_{\mathrm{ijk} l}=a_{i)(j)(1}$ and for the effective grain inhomogeneity of the ellipsoidal form it is possible to obtain

$$
\begin{gathered}
\mathrm{a}_{\mathrm{ijkl}}=-(1 / 4 \pi) \int \mathrm{n}_{\mathrm{ij}} \Lambda_{\mathrm{kl}}^{-1} \mathrm{~d} \Omega \\
\Lambda_{\mathrm{ij}} \equiv \mathrm{c}_{\mathrm{ijk} \mathrm{k}} \mathrm{n}_{\mathrm{kl}}
\end{gathered}
$$

The normal direction in spherical coordinates has the form

$$
\begin{aligned}
& \mathrm{n}_{1}=\left(1 / 1_{1}\right) \sin \vartheta \cos \varphi, \\
& \mathrm{n}_{2}=\left(1 / 1_{2}\right) \sin \vartheta \sin \varphi, \\
& \mathrm{n}_{3}=\left(1 / 1_{3}\right) \cos \vartheta, \\
& \mathrm{d} \Omega=\sin \vartheta \mathrm{d} \vartheta \mathrm{d} \varphi .
\end{aligned}
$$

The expression (2.12) can be written in a more compact way (Shermergor, 1977)

$$
c^{*}=\left\langle(c+b)^{-1}\right\rangle^{-1}-b, b=-c-g^{-1}
$$

By choosing the comparison tensor $c^{c}$ equal to zero and infinity respectively we obtain the approximation of Voigt and Reuss, that is $c^{*}=\langle c\rangle$ and $c^{*}=\left\langle c^{-1}\right\rangle^{-1}$ the Hill's boundaries:

$$
\langle c\rangle<\mathrm{c}^{*}<\left\langle\mathrm{c}^{-1}\right\rangle^{-1}
$$

Selecting the stiffness tensor of a body of comparison equal $\langle c\rangle$ or $\left\langle c^{-1}\right\rangle^{-1}$, we shall obtain the solution of Hashin - Shtrikman approximation (Hashin and Shtricman 1962)

In reference (Kuzmenko and oth., 1983) the above approximation is applied to anisotropic elastic textured polycrystals and in reference (Shermergor and oth., 1991) 
to textured rocks. Piezoelectric properties of polycrystals consisting crystallites of high symmetry with an elementary texture were considered in work (Valter and oth., 1991, 1993).

\section{THE DYNAMIC EFFECTIVE CHARACTERISTICS}

The dynamic effective characteristics are understood as the same characteristics described in the previous section, but now it is assumed, that they depend on the wave vector. In a more general case it appears that they depend on the frequency as well. In this case the dispersion appears to be not only a spacial one, but also a time and frequency dispersion. However, the time dependence dispersion in the following discussion will be neglected. If for a given material and in a given range of frequencies it become essential, it can be taken into account by the additive value to the parameter of the scattering of waves.

The dynamic characteristics of a material essentially depend on a type of inhomogeneities of the material as well as on the character of waves. At a smooth change of physical properties of a medium the binary correlation functions of the Gauss type are used, for which, at a transition through zero, the derivative does not jump Tatarskij, 1961, Klyatskin, 1980). For the media with sharp borders between the grains of heterogeneities the correlation functions are used, for which the derivative at the transition through zero has a jump. An example of the first type of media is air, in which the heterogeneity is stimulated by thermal fluctuations.

By medium of the second type we mean polycrystals, rocks and composites. Below only materials of the second type are considered. We consider only hard materials with microheterogeneities. The elementary correlation function, taking into account a sharp border between the grains and the matrix is an exponential function (Lifshits and Parchomovskii 1950). However, the use of the $\varphi(\vec{r})=\exp (-r / a)$ type does not permit to take a texture into account. The simplest generalization of this dependence on the case of macroscopically anisotropic media is (Fokin and Shermergor, 1994, 1995)

$$
\varphi(\vec{r})=\exp (-r / a) \cos (\vec{b} \vec{r}), \vec{r} \equiv \vec{r}_{1}-\vec{r}_{2}
$$

We shall emphasize, that such dependence covers also the even property of the correlation function.

Qualitatively the dynamic characteristics for transverse waves, propagating in a heterogeneous medium weakly depend on the type of the transverse waves - either electromagnetic or elastic ones. Scalar waves have the same dynamic characteristics. Therefore, for definiteness, we shall consider monochromatic electromagnetic waves, for which the vector of intensity of the electrical field has a form

$$
\vec{E}(\vec{r}, t)=\vec{E}(\vec{r}) \exp (-i \omega t)
$$

The wave equation in the absence of sources of waves takes a form

$$
\begin{gathered}
\mathrm{LE}=0, \\
\mathrm{~L} \equiv \nabla^{2} \mathrm{I}+\mathrm{k}_{0}^{2} \varepsilon(\overrightarrow{\mathrm{r}}), \mathrm{k}_{0}^{2}=\omega^{2} / \mathrm{c}_{0}^{2}
\end{gathered}
$$


Averaging the material equation of the relationship between the electrical induction $\vec{D}$ and intensity of the electrical field $\overrightarrow{\mathrm{E}}$, we obtain

$$
\langle\overrightarrow{\mathrm{D}}\rangle=\langle\varepsilon \overrightarrow{\mathrm{E}}\rangle=\varepsilon^{*}\langle\overrightarrow{\mathrm{E}}\rangle
$$

Here $\varepsilon^{*}$ is the integrated operator, covering the nonlocal property of the medium.

The wave equation for the average fields is

$$
\mathrm{L}^{*}\langle\overrightarrow{\mathrm{E}}\rangle=0, \mathrm{~L}^{*} \equiv \nabla^{2} \mathrm{I}+\mathrm{k}_{0}^{2} \varepsilon^{*}, \mathrm{k}_{0}=\omega / \mathrm{c}
$$

The average intensity of the electrical field for a monochromatic wave is expressed through the wave vector of the effective medium $\overrightarrow{\mathrm{k}}^{*}$ :

$$
\langle\overrightarrow{\mathrm{E}}\rangle=\overrightarrow{\mathrm{E}}_{0} \exp \left(\mathrm{i}^{*} \overrightarrow{\mathrm{r}}\right)
$$

Alongside with the micrononuniform medium we shall consider a similar uniform medium equivalent to it (medium of comparison). Quantities related to such a medium shall be designated by the index c. We shall define the Green function for the medium of comparison by the equation

$$
\mathrm{L}^{\mathrm{c}} \mathrm{H}^{\mathrm{c}}=-\mathrm{I} \delta(\overrightarrow{\mathrm{r}}), \mathrm{L}^{\mathrm{c}} \equiv\left(\nabla^{2}+\mathrm{k}_{\mathrm{c}}^{2}\right) \mathrm{I}, \mathrm{k}_{\mathrm{c}}^{2} \equiv \varepsilon_{\mathrm{c}} \mathrm{k}_{0}^{2}
$$

Then considering only pair interactions for the operator of the effective permittivity we obtain

$$
\varepsilon^{*}=\langle\varepsilon\rangle+\mathrm{k}_{0}^{2}\left\langle\varepsilon^{\prime \prime} \mathrm{H}^{\mathrm{c}} \varepsilon^{\prime \prime}\right\rangle
$$

Here we designate $\varepsilon^{\prime \prime}(\overrightarrow{\mathrm{r}}) \equiv \varepsilon(\overrightarrow{\mathrm{r}})-\varepsilon_{\mathrm{c}}$. The approximation (3.8) is called the Bourret approximation (Bourret $(1962,1965)$ ). In the majority of cases it is convenient for the permittivity of the body of comparison to choose its average magnitude: $\varepsilon_{\mathrm{c}}=\langle\varepsilon\rangle$ and in this case $\varepsilon^{\prime \prime}=\varepsilon^{\prime}$.

For the homogeneous equation to take place it is required that the corresponding determinant be equal to zero

$$
\operatorname{det}\left\|\mathrm{k}^{2} \delta_{\mathrm{ij}}-\mathrm{k}_{0}^{2} \varepsilon_{\mathrm{ij}}\right\|=0
$$

Calculating the determinant, we obtain a dispersion equation relative to the dimensionless effective wave vector:

$$
\begin{gathered}
\mathrm{x}^{2}-\mathrm{q}^{2} \bar{\varepsilon}^{*}(\overrightarrow{\mathrm{x}}, \mathrm{q})=0 \\
\overrightarrow{\mathrm{x}} \equiv \mathrm{ak}, \mathrm{q} \equiv \mathrm{ak}_{\mathrm{c}}, \bar{\varepsilon} \equiv \varepsilon / \varepsilon_{\mathrm{c}}
\end{gathered}
$$

The tensor $\varepsilon^{*}(\overrightarrow{\mathrm{x}}, \mathrm{q})$ presents the Fourier transform $\varepsilon^{*}(\overrightarrow{\mathrm{r}}, \omega)$ of a nucleus of the integrated operator $\varepsilon^{*}$. Calculations result in the following

$$
\begin{gathered}
\mathcal{E}^{*}(\overrightarrow{\mathrm{x}}, \mathrm{q})_{\text {eff }}(\overrightarrow{\mathrm{x}}, \mathrm{q})=\langle\varepsilon\rangle+\overrightarrow{\mathrm{D}}_{\varepsilon}^{*} \mathrm{~F}(\overrightarrow{\mathrm{x}}, \mathrm{q}) \\
\mathrm{F} \equiv(1 / 8 \pi) \varphi * \mathrm{Q}_{\mathrm{c}}, \mathrm{Q}_{\mathrm{c}}(\overrightarrow{\mathrm{x}}, \mathrm{q})=\mathrm{q}^{2} /\left(\mathrm{x}^{2}-\mathrm{q}^{2}\right) \\
\mathrm{F}=\mathrm{q}^{2}\left[\mathrm{x}^{2}+\mathrm{b}^{2}-(\mathrm{q}+\mathrm{i})^{2}\right] /\left\{\left[\mathrm{x}^{2}+\mathrm{b}^{2}-(\mathrm{q}+\mathrm{i})^{2}\right]^{2}-(2 \overrightarrow{\mathrm{x}} \overrightarrow{\mathrm{b}})^{2}\right\}
\end{gathered}
$$


The asterisk in (3.13) is symbol of convolution. Once the complex value of the wave vector $\vec{x}$ is found we find normalized dynamic parameters of a micrononuniform medium: the parameter of scattering, phase and group velocity of the propagation of transversal waves.

$$
\begin{gathered}
\bar{\gamma} \equiv \mathrm{a} \gamma=2 \mathrm{x}_{2}, \mathrm{x} \equiv \mathrm{ak}^{*}=\mathrm{x}_{1}+\mathrm{ix}_{2} \\
\overline{\mathrm{v}} \equiv \mathrm{q} / \mathrm{x}_{1}=\mathrm{v}^{*} / \mathrm{v}_{\mathrm{c}}, 1 / \overline{\mathrm{c}} \equiv \mathrm{dx}_{1} / \mathrm{dq}=\mathrm{v}_{\mathrm{c}} / \mathrm{c}, \mathrm{v}_{\mathrm{c}}=\mathrm{c}_{0} \varepsilon^{-1 / 2}
\end{gathered}
$$

In reference (Fokin and Shermergor, 1989a) the above approximation is applied to the problem of elastic wave propagation and in reference (Shermergor and Fokin, 1986, Fokin and Shermergor 1989b, 1989c) to electromagnetic ones.

\section{RESULTS AND DISCUSSION}

In earlier works on the use of random fields to calculate the dynamic parameters of structurally heterogeneous media the Born approximation was used. It made possible to calculate the factor of scattering, the phase and group velocities by a given correlation function of material parameters in the case of the long wave approximation that is when the wavelength is much greater then the average size of inhomogenities. Applied to solid microinhomogeneous materials the corresponding calculation were published in (Lifshits and Parchomovsii, 1950, Shermergor, 1977, Usov and Shermergor, 1978). Later this branch of heterogeneous medium mechanics was developed in two ways. The first one consisted in searching for a more exact and complicated model of heterogeneous materials, while the second one tried to use higher approximations of the random field theory whereas relatively simple expressions of the binary correlation function of material parameters was used. The latter branch has been successfully developed by Bourret (Bourret, 1962, 1965) and applied to the case of scalar and dielectric waves in heterogeneous isotropic media by Karal and Keller (Karal and Keller, 1964). This allowed to calculate dynamic characteristics of heterogeneous material in the whole range of wavelengths using a spherically symmetric correlation function of material parameters $\varphi(\vec{r})=\exp (-\mathrm{r} / \mathrm{a})$. The same approach was used by Stanke and Kino (Stanke and Kino, 1984) to calculate the attenuation factor and the phase velocity of the transverse and longitudinal elastic waves in the monophase nontextured polycrystalline media. They have done numerical calculations and obtained the relationships between the scattering factor and the phase velocity on the normalized wave number $\mathrm{q}=2 \pi \mathrm{a} / \lambda$, where $\lambda$ is the wavelength and $\mathrm{a}$ is the space scale of correlation.

Unlike the Born's approximation the Bourett's approach considers the multiple scattering of the wave passing through a stochastic medium. Here mathematical difficulties do not allows to go beyond the pair correlations as it was possible when calculating static characteristic. Unlike the Born's approximation the Bourret's approach leads to a square equation (3.12). The solution of this equation gives two roots. In case of the long wave approximation one of them results in the same relationship between the scattering factor and the phase velocity as the Born's approach does. This wave is referred to us a real one. The second root gives in the long wave range such a large value for the scattering factor that the corresponding wave practically cannot propagate in a micrononuniform material. Such kind of waves is usually called a virtual one. 
Figure 1 shows the relationship between the real and virtual waves scattering factors in the whole range of wavelengths for a textured medium described by the correlation factor (3.1) at the parameter $b=1$ and the dimensionless dispersion $\log D=4$. In fact, in the range of long waves $(q<<1)$ and even middle waves $(q \sim 1)$ the scattering factor for the virtual wave is much greater than that for the real wave and thus in this range the virtual wave can be totally neglected. In case of ultrashort waves $(q>>1)$ the situation is quite different. We can see from the graph that starting with $\log q=2$ both factors coincide and therefore in this region one should speak of the superposition of the real and virtual waves.

To estimate the influence of the dispersion value on the scattering factor a family of corresponding curves as a function of the wave number $\mathrm{q}$ for the isotropic correlation function $(b=0)$ is given in Figure 2. If we approximate the dependence of the scattering factor on the wave number by the exponential relationship $\gamma=$ const $\mathrm{q}^{\mathrm{p}}$, then for the assumption of long waves we get Raighley's Law, i.e. $p=4$, while for ultrashort waves

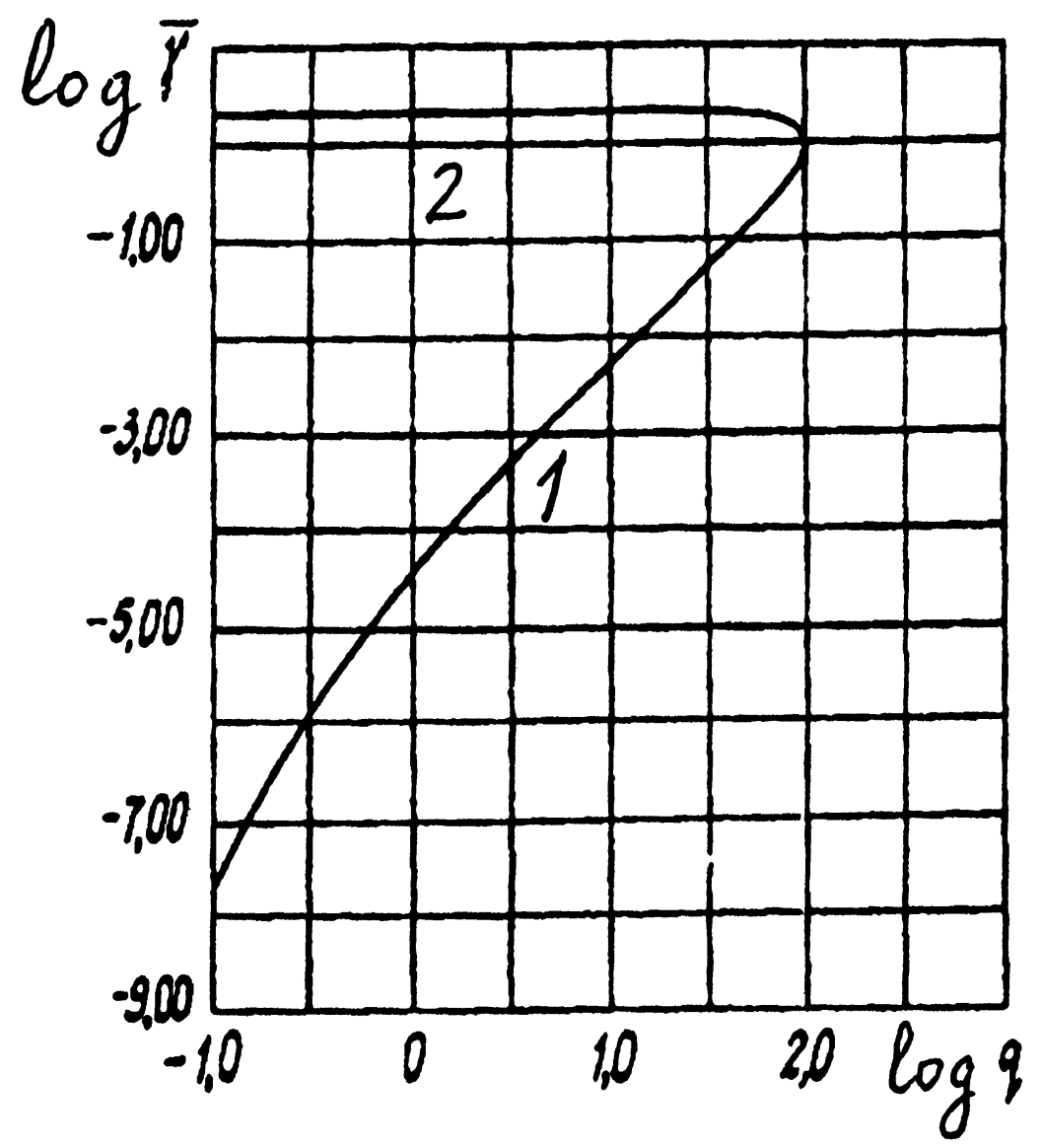

Figure 1 Normalized scattering index $\bar{\gamma}, 1$ - real wave, 2 - imaginary wave. $\ln D_{e}=-4$. 


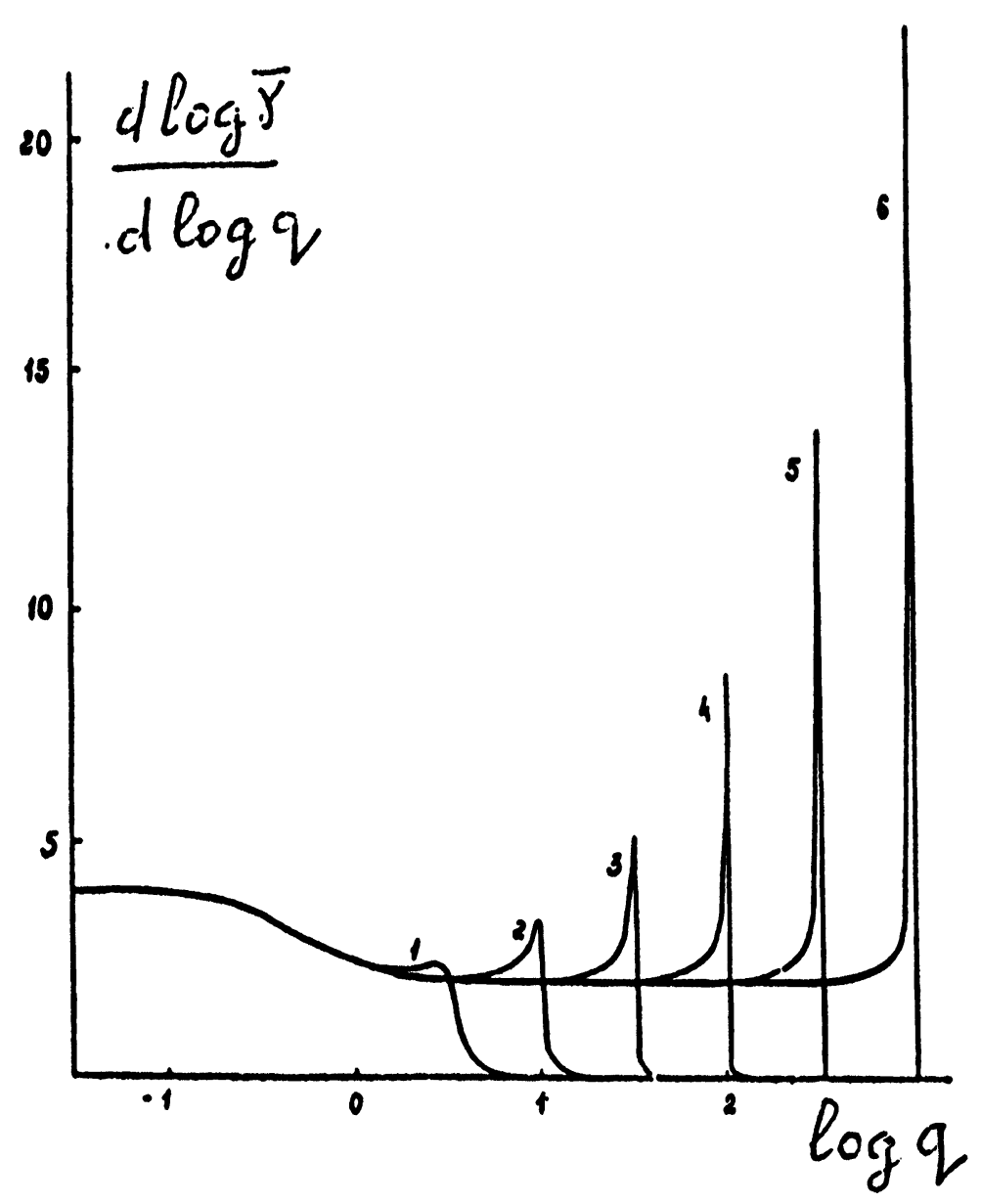

Figure 2 The parameter $\mathrm{p}=\mathrm{d} \log \bar{\gamma} / \mathrm{d} \log \mathrm{q}$ as a function of the logarithm of dimensionless wave number. The numericals indicate the absolute values of $\bar{D}_{\varepsilon}$.

we get the approximation of geometrical optics $p=0$, and for the intermediate region it is $p=2$. One can easily see, that in the transition regions the exponential approximation is not valid and therefore the peaks on the curves have no physical sense. Here we stress two features: the first one is that the lower is the dispersion of material characteristics the greater is the number of the wave numbers for which the intermediate asymptotic $(p=2)$ is valid. For a sufficiently great dispersion the intermediate asymptotic can be absent at all. The second feature consist in the fact that with decreasing dispersion the left bound of the area of existence for the geometrical asymptotic $(p=0)$ is shifting towards the shorter wavelength region. And as one should have expected for homogeneous medium scattering does not take place at all: $\gamma \rightarrow 0$.

The dependence of the phase and group velocities of the real wave propagation on the wave number $\mathrm{q}$ is shown in Figure 3, while the same dependencies for the virtual 


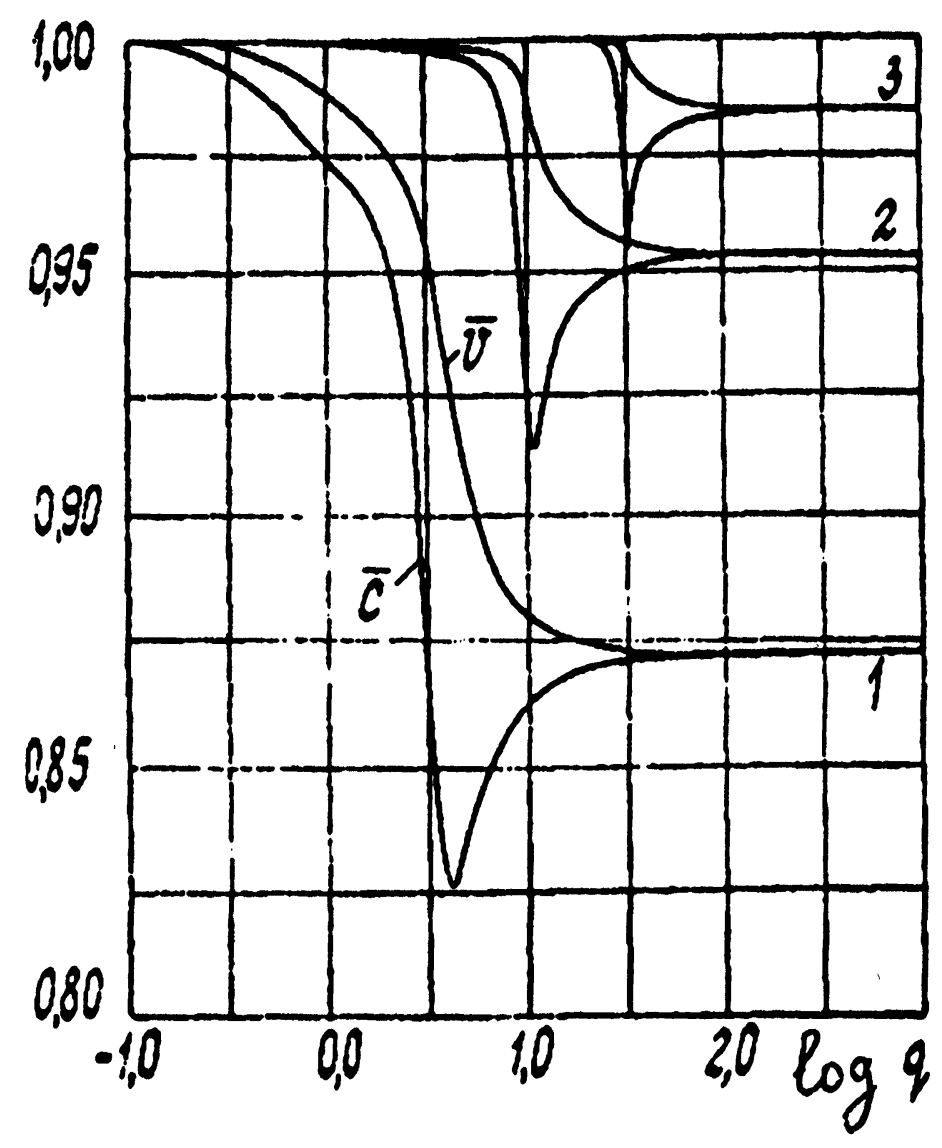

Figure 3 Renormalized group velocity $\bar{c}$ and phase velocity $\bar{v}$ of the real wave as a function of the dimensionless frequency. $\mathrm{b}=1$.

$1-\ln D_{e}=-1$

2- $\ln D_{e}=-2$

3- $\ln D_{e}=-3$

wave are given in Figure 4. The curves for the virtual wave should have a mirror symmetry with respect to the axes $\bar{c}=\bar{v}=1$ of the graphs for the real wave curve. In order to take into consideration the contribution of the parameter $b$ which takes into account the deviation of the correlation function from the spherical symmetry the relationship between the waves propagation velocities and the wave numbers Figure 3 is given for $b=1$, while Figure $4-$ for $b=0$.

It can be seen that when $b$ is increasing the discontinuity on the group velocity curves become smoother. Note, that while the phase velocity in the above case of electromagnetic waves monotonously decreases with the increase of $q$, one can observe a sharp minimum for the group velocity. And this minimum occurs in the range of the transition from short waves to ultrashort ones. The second distinct feature is that when the dispersion of the material parameter $\overline{\mathrm{D}}_{\varepsilon} \equiv\left\langle(\varepsilon-\langle\varepsilon\rangle)^{2}\right\rangle^{1 / 2} /\langle\varepsilon\rangle$ (here it is dispersion of 


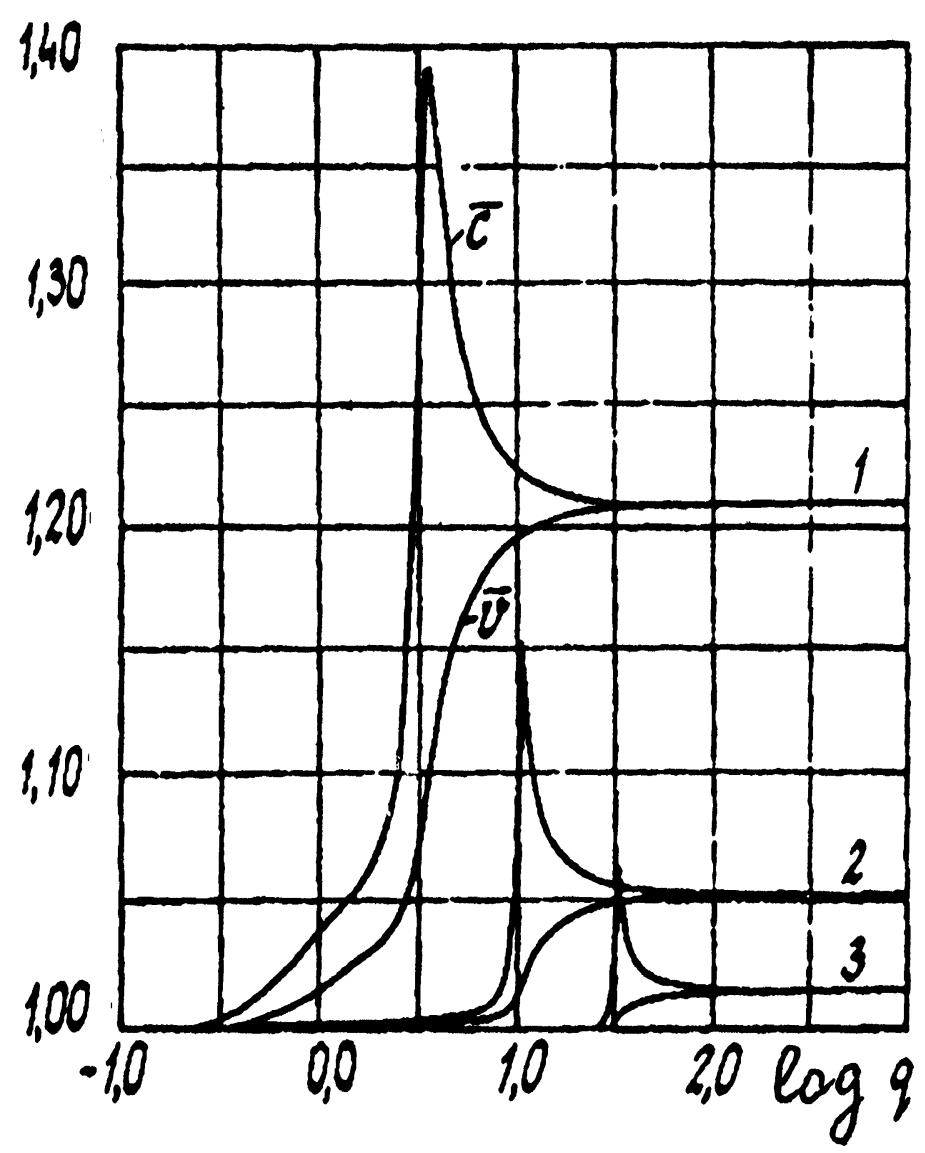

Figure 4 Renormalized group velocity $\bar{c}$ and phase velocity $\bar{v}$ of the real wave as a function of the dimensionless frequency. $\mathrm{b}=0$. Curves as in Figure 3

dielectric permiability) decreasing, the physical dispersion, i.e. the dependence of the wave propagation velocity on the frequency, decreases (when $\bar{D}_{\varepsilon} \rightarrow 0, c_{\min } / \mathrm{c}_{\max }$ $\rightarrow 1)$. The sharp discontinuity on the curve describing the dependence of the group velocity on the wave number occurs at any infinitely small value of $\bar{D}_{\varepsilon}$. This discontinuity is in agreement with the sharp change of the curve behavior for the scattering factor in the region of $\log q=2$ shown in Figure 1. Similar results for the scattering factor were obtained by Stanke and Kino (1984) for polycrystalline aluminum. Here too the transfer to the geometrical optics limit starts with the value of $\log q=2$.

\section{References}

Bourret R. S. (1962). Propagation of Randomly Perturbed Fields. Canad. J. Phys., Vol 40. P. 782. Bourret R. S. (1965). Fiction Theory of Dynamical Systems with Noisy Parameters. Canad. J. Phys., Vol 43. P. 619.

Bunge, H. J. (1993). Texture Analysis in Material Sciences, Cuvillier. Göttingen 330p. 
Chernov L. (1960). Wave Propagation in a Random Medium. McGraw-Hill. Publ. Co., New York.

Fokin A. G. and Shermergor T. D. (1989). The Theory of Elastic Wave Propagation in Inhomogeneous Media. Phys. stat. sol. (b). V. 153, No. 1. P. 89-100.

Fokin A. G. and Shermergor T. D. (1989). Theory of Propagation of Electromagnetic Waves in Transparent Ferroelectric Ceramics. Ferroelectrics, V. 90, No. 1. P. 15-21.

Fokin A. G. and Shermergor T. D. (1989). Electromagnetic Wave Scattering in Inhomogeneous Media with Spatial Dispersion. Phys. stat. sol. (b). V. 15, P. 367-376.

Fokin A. G. and Shermergor T. D. (1994). Dynamic Characteristics of Multicomponent Polycrystals and Composites. Thesis's of Lectures of 5th International Conference. "Lasers in science, engineering and medicine" 22 September 1994. Suzdal. Moscow, P. 26-30.

Fokin A. G. and Shermergor T. D. (1995). Influence of Near Order on Middle Field in a Stochastic Media. Zh. Exper. Theor. Fiz., V. 107, No. 1. P. 111-118.

Ginzburg V. L. (1967). Propagation of Electromagnetic Waves in Plasma. Izd. Nauka, Moscow. (in Russian).

Hashin Z. and Shtricman S. (1962). On some Variational Principles in a Anisotropic and Nonhomogeneouse Elasticity. Journal Mech. Phys. Solids. Vol. 10, No. 4, P. 335.

Karal F. C. Jr. and Keller J. B., (1964). Elastic, Electromagnetic, and Other Waves in a Random Medium. J. Math. Phys., Vol. 5. P.537.

Klyatskin V. I., (1980). Stochastic Equations and Waves in Randomly Inhomogeneous Media. lzd. Nauka, Moscow, (in Russian)

Kuzmenko Yu. V., Korneev V. I. and Shermergor T. D. (1983). Effective Elastic Properties of Axial Textures. Materials Science and Engineering. Vol 58, No. 5, P. 169-174.

Lifshits I. M. and Parchomovskii G. D. (1950). On Theory of Propagation of Ultrasonic Waves in Polycrystals. Zh. Exper. Theor. Fiz., V 20, No. 2, P. 175.

Monin A. S., and Jaglom A. M. (1965). Statistical Hydromechanics, Mechanics of Turbulence. Part 1. lzd. Nauka. Moscow (in Russian)

Monin A. S., and Jaglom A. M., (1967). Statistical Hydromechanics Mechanics of Turbulence. Part 2. lzd. Nauka, Moscow (in Russian)

Rytov S. M., Kravtsov Yu. A. and Tatarsky V. I. (1978). Introduction of Statistical Radiophysics. Part II, Random Fields. lzd. Nauka, Moscow (in Russian)

Shermergor T. D., (1977). The Elasticity Theory of Microheterogeneous Media. lzd Nauka, Moscow (in Russian)

Shermergor T. D. and Fokin A. G. (1986). Scattering of Electromagnetic Waves in Ferroelectrics Ceramics. Ferroelectrics. No. 1. P. 43-49

Shermergor T. D. and Dikarev A. V. (1991). Stochastic Resonance under Propagation of Electromagnetic Waves in a Three-Dimensional Random Medium. Physica status solidi (b). V. 168. No. 1, P. 305.

Shermergor T. D. Nikitin A. N., Walter K., Voitus V., Ivankina T. I. and Jakovlev V. B. (1991). Calculation of the Effective Elastic Moduli of Textured Piezoelectric Rocks. lzv. Acad. Nauk USSR. Phys. Earth. No. 12, P. 94-103

Shermergor T. D., Fokin A. G. and Dikarev A. V. (1991). Stochastic Resonance of Waves Propagated in Fully Disordered Three-Dimensions medium. Dokl. Acad. Nauk USSR, Vol. 320, No. 5, P. 1063

Shermergor T. D., Fokin A. G. and Dikarev A. V., (1992). Stochastic Resonance Under Wave Propagation in Disordered Media. Ferroelectrics, V. 131. P. 97.

Stanke F. E. and Kino G. S. (1984). A Unified Theory for Elastic Wave Propagation in Polycrystalline Materials. J. Accoust. Soc. Amer. Vol. 75, P. 665.

Tatarskij V. I. (1961). Wave Propagation in Turbulent Media. McGraw-Hill Publ. Co., New York.

Walter K., Ivankina, T. I., Nikitin A. N. Voitus V., Shermergor T. D. and Jakovlev V. B. (1991). The Determination of Effective Physical Characteristics of Anisotropic Geomaterials with Help of Texture Analysis. Dokl. Acad. Nauk USSR, V. 319, No 2, P.310.

Walter K., Nikitin A. N., Voitus V., Shermergor T. D. and Jakovlev V. B. (1993). The Determination of Effective Electroelastic Constants of Polycrystalline Textured Rocks. Physics of Earth. No 6, P. 83-88. 